\title{
Kinematic Analysis of Forehand Stroke Technology of the World Excellent Male Tennis Player Nadal
}

\author{
GuangWen Song ${ }^{1, a^{*}, J_{i H e Z h o u}^{2, b} \text { and JunGuo }}{ }^{3, c}$ \\ ${ }^{1}$ Chengdu Sport University , Chengdu, Sichuan Province, China \\ ${ }^{2}$ Chengdu Sport University, Chengdu, Sichuan Province, China \\ ${ }^{3}$ Chengdu Sport University, Chengdu, Sichuan Province, China

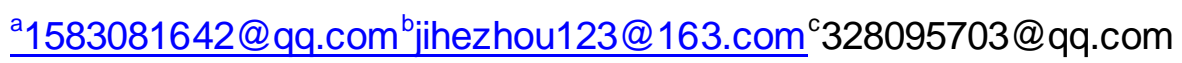

\begin{abstract}
Keywords:Tennis; Forehand Stroke Technology; Kinematics
Abstract.By filming the excellent male athlete Nadal's live game video in the 2014 China Open, the thesis analysis Nadal's forehand stroke technique in virtue of three-dimensional photograph analytic method, selecting the standard video of Nadal's baseline forehand topspin. It is aimed at revealing the kinematic characteristics of the world's excellent male tennis player's forehand stroke technology and to provide theoretical basis for athletes to correct errors and for coaches to guide training.
\end{abstract}

\section{Introduction}

Tennis, with aerobic and anaerobic exercise carried out alternately but mainly aerobic exercise, is a kind of skill dominating, net separated, antagonistic and fashionable sanatory sport. Forehand technique is the entry-level technology and also one of the basic technologies for each player in tennis. During the game, about $70 \%-80 \%$ balls was forehand hitting. Nadal's powerful forehand contributed a lot to his achievements for 8 consecutive years in Monte Carlo. Researches on tennis absorbed themselves in the upper limbs and torso. However in recent years, due to the improved technology, the hitting power of tennis comes mainly from the lower limbs while pushing against the grand. By using the 3-D Signal TEC V2.0HDC software developed by Beijing Senmiaoxing Science and Technological Development Co., Ltd to analyzing the world excellent athlete's live game video in the 2014 China Open, some valid data of the athlete's upper and lower limbs and torso in different period can be obtained, and these data have much significance for improving tennis technology and tennis teaching.

\section{Methods}

2014 China Open employed two made-in-Japan JVC9800 cameras (shooting frequency: 50fpS) to shoot the entire forehand stroke of Nadal. The cameras' horizontal height is 1.25 meters. No.1 camera was fixed right behind the side line of the tennis court, and No.2 camera was taken right in front of the base line, showing an included angle of about $45^{\circ}$ between main optical axes of the two cameras. Europe Dempster (16 links, 21 joints parameter) was chosen as the mannequin. The shot videos was analyzed by the 3-D Signal TEC V3.2HDC software developed by Beijing Senmiaoxing Science and Technological Development Co., Ltd, frame by frame. For the study need, the analysis added two projection angles of the racket top and shoulder hip part. The received original data was smoothed by low-pass digital filtering method and the cutting frequency reached to $8 \mathrm{~Hz}$.

\section{Results}

Nadal's forehand stroke use semi-open stance play. The whole forehand consist of 4 parts: shoulder turning, back-swing, hitting the ball and the follow-swing on the upper body. During the entire operation, the body rotated about $180^{\circ}$ from the end of back-swing to the end of hitting the ball. At the end of back-swing, Nadal made his left and right hip angles to $130.8^{\circ}$ and $160.6^{\circ}$ respectively, 
left and right knee angles of $132.3^{\circ}$ and $141.8^{\circ}$, which illus trates that Nadal's hip and knee are in flexed position. His left shoulder angel of $74.8^{\circ}$ and left elbow angle of $131.9^{\circ}$ showed the athlete's arms' extended state relatively to the torso at the end of the back-swing. In the case of extending the same distance, the left shoulder angle and the left elbow angle is inversely proportional. The bigger the left elbow angle is, the smaller the left shoulder angle, and the smaller the left elbow angle is, the bigger the left shoulder angle. During the back-swing, the speeds of his left shoulder, left elbow, left wrist joint, and the center of gravity were $0.78 \mathrm{~m} / \mathrm{s}, 1.94 \mathrm{~m} / \mathrm{s}, 4.86 \mathrm{~m} / \mathrm{s}$ and $2.26 \mathrm{~m} / \mathrm{s}$ respectively. Combined with the live game video, during the period from Nadal's shoulder turning to the end of the back-swing, Nadal's body's center of gravity is in a dynamic process of falling, and the direction of the linear velocity of the center of gravity is mainly downward. The downward linear velocity of body's center of gravity and the back-swing linear velocity caused by the left shoulder and left elbow jointed action, and formed the left wrist linear velocity of Nadal. However, during the period from hitting the ball to the end of the finish-swing, Nadal's body's center of gravity is in a dynamic process of rising. The image of body's center of gravity in the whole forehand stroke(excluding hops operation) was crest - trough - crest type, which was not only complied with the tennis forehand objective laws, but also reflected strongly that Nadal could make a good topspin. Nadal made the shoulder-hip angle of $-38.8^{\circ}$ at the end of back-swing and $8.6^{\circ}$ at the moment of hitting the ball, with a rotation angle of $47.4^{\circ}$ between shoulder and hip in this process. During the period of swing and hit the ball, from the live game video, Nadal's body rotated about $180^{\circ}$ from the end of the back-swing to the end of hitting the ball, and this data reflected the large range of Nadal's body rotation. Before racket hitting the ball, the torso was in a certain tension, and accessed larger elastic potential energy, which played an important role in the followed hitting. It took $0.73 \mathrm{~s}$ from shoulder turning to the moment when the ball touched the racket, wherein $0.62 \mathrm{~s}$ for back-swing and $0.11 \mathrm{~s}$ from the end of back-swing to the moment when the ball touched the racket, which shows that the time for back-swing is long and the speed is slow, while that the time for forward-swing and hitting is short and the speed is faster, which can increase the speed of the ball by enlarge the inertia of the racket. While, at the moment of stroke, the speeds of Nadal's left shoulder, left elbow, left wrist, and left hand were $2.21 \mathrm{~m} / \mathrm{s}, 4.58 \mathrm{~m} / \mathrm{s}, 6.27 \mathrm{~m} / \mathrm{s}$, and $7.50 \mathrm{~m} / \mathrm{s}$ respectively. It can be seen that the speed of wrist is relatively larger than the shoulder and elbow's. While hitting topspin, the wrist was not fixed. At the moment of hitting, it is the elbow's inward rotation drive the wrist to shake that to make the topspin. At the end of follow-swing, Nadal's racket was on the top of the head, while other athletes were finished with their rackets under shoulders or more slightly below. At this moment, the left shoulder angle, the left elbow angle and the left wrist angle are $113.4^{\circ}, 87.9^{\circ}$ and $98.2^{\circ}$ respectively, which is different from other athletes. It increased the friction time and distance of the racket and the ball, which can be of absolutely reference value on how to make a super powerful topspin.

\section{Conclusion}

In conclusion, Nadal's forehand stroke use semi-open stance play. During the entire operation, the body rotated about $180{ }^{\circ}$ from the end of back-swing to the end of hitting the ball, with a rotation angle of $47.4^{\circ}$ between shoulder and hip. The technology data are: (1)Back-swing:At the end of back-swing, Nadal made his left and right hip angles of $130.8^{\circ}$ and $160.6^{\circ}$ respectively, the horizontal shoulder-hip projected angle of $38.8^{\circ}$, and the left shoulder angel and left elbow angle of $74.8^{\circ}$ and of $131.9^{\circ}$ respectively. (2) Hitting the ball: At the moment of stroke, the horizontal shoulder-hip projected angle is $8.6^{\circ}$, and the speeds of Nadal's left shoulder, left elbow, left wrist, and left hand were $2.21 \mathrm{~m} / \mathrm{s}, 4.58 \mathrm{~m} / \mathrm{s}, 6.27 \mathrm{~m} / \mathrm{s}$, and $7.50 \mathrm{~m} / \mathrm{s}$ respectively. (3)the follow-swing: At the end of the follow-swing, the left shoulder angle, the left elbow angle and the left wrist angle are $113.4^{\circ}, 87.9^{\circ}$ and $98.2^{\circ}$ respectively. In order to make a super powerful topspin, Nadal's body's center of gravity had relatively fewer up and down, and the friction time and distance of the racket and the ball were relatively long. At the same time, the elbow's inward rotation drive the wrist to shake to make the topspin. Nadal's follow-swing differs a lot from other excellent athletes, and this motion is not suggested to imitate. 


\section{References}

[1] Li Yifeng. Batting rhythm in the tennis teaching of graduate thesis[D]. Wuhan Sports Institute, 2 007.

[2] Wang Huifang, Zhou Jihe, Gao Zhan Le, Li Yirong. Enquist tennis forehand topspin skill kinem atic analysis of [A]. Chinese Association of biomechanics in Sports Association. The Sixteenth Nati onal Sports Biomechanics Symposium (CABS 2013) [C]. proceedings of China Association of bio mechanics in sports: branch, 2013:2

[3] Hu Qiquan biomechanical nalysis of China's outstanding young tennis players forehand topspin [D]. Beijing Sport University, 2008.

[4] Sun Yanming, Chang Chunfang. Kinematics tennis forehand topspin technical characteristics an alysis [J]. Journal of Capital Institute of Physical Education, 2014,02:189-192.

[5] Ma Yanhui. Biomechanical malfunction analysis of forehand tennis[J]. examination weekly, 20 07,39:94-95.

[6] Guo Jian. Tennis forehand technology analys is and biomechanical evaluation [J]. sports science and technology journal, 2009,12:53-55 